Supporting Information

\title{
Protective Mechanism of Rice-Derived Lipids and Glucosylceramide in an In Vitro Intestinal Tract Model
}

\author{
Shinji Yamashita ${ }^{1}$, Michiru Soga $^{1}$, Ephantus Nguma $^{1}$, Mikio Kinoshita $^{1 *}$, and Teruo Miyazawa ${ }^{2}$ \\ ${ }^{1}$ Department of Life and Food Sciences, Obihiro University of Agriculture and Veterinary Medicine, \\ Obihiro 080-8555, Japan \\ ${ }^{2}$ Food and Biotechnology Platform Promoting Project, New Industry Creation Hatchery Center (NICHe), \\ Tohoku University, Sendai 980-8579, Japan
}

*Corresponding author

Email: kinosita@obihiro.ac.jp, Phone:+81-155-5445, Fax: +81-155-49-5593 
a

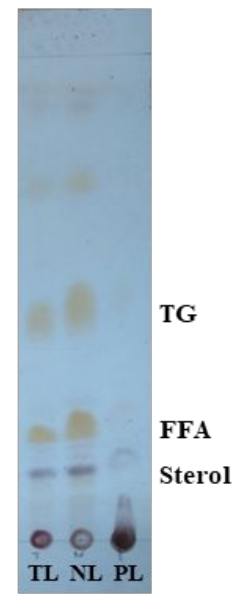

b

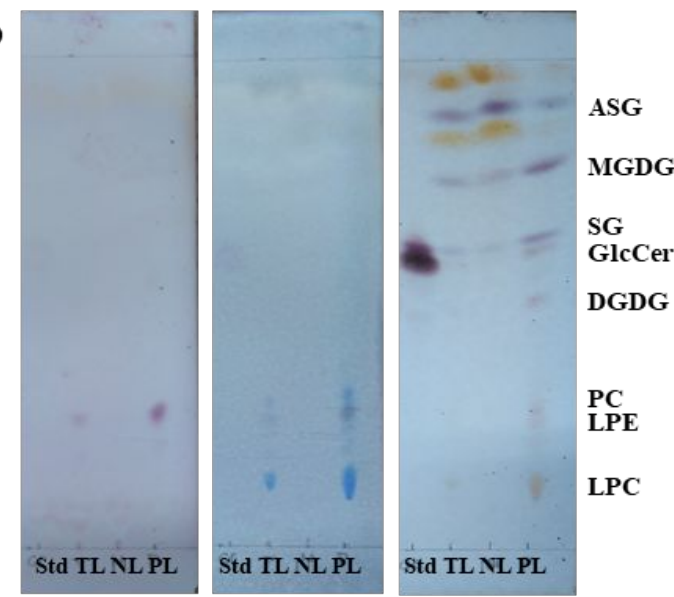

c

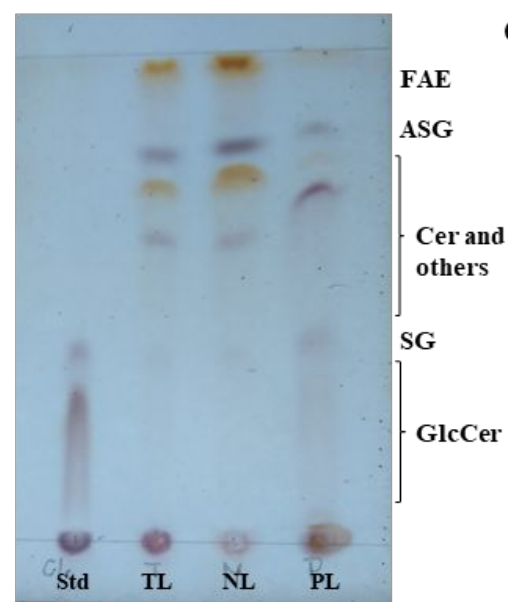

d

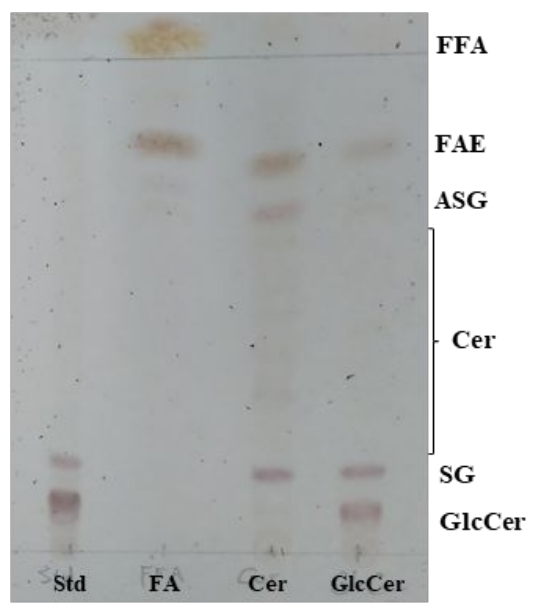

Figure S1. Profiles of lipid rich fractions from rice and sake lees determined using thin layer chromatography. a, Neutral lipid profiles of TL, NL, and PL from rice. Mobile phase: hexane/diethylether/acetate (80/20/1, v/v/v). Detection: 50\% sulfate; b, Polar lipid profiles of TL, NL, and PL from rice. Mobile phase: chloroform/methanol/water (65/25/4, v/v/v). A GlcCer and SG mixture was used as the Std. Detection (left, central, and right panels): ninhydrin reagent (for amino group), Dittmer reagent (for phosphorus), and 50\% sulfate, respectively. c, Alkaline-stable lipid profiles of TL, NL, and PL from rice. Sphingolipids and sterols were detected after degradation of glycerolipids by mild alkalinetreatment. Mobile phase: chloroform/methanol $(95 / 12, \mathrm{v} / \mathrm{v})$. Detection: $50 \%$ sulfate. d, Lipid profiles of FA, Cer-rich, and GlcCer-rich fractions from sake lees. Mobile phase: chloroform/methanol (95/12, v/v). Detection: $50 \%$ sulfate.

ASG, acylsterylglycoside; Cer, ceramide; DGDG, diglycosyldiacylglycerol; FA, fatty acid; FAE, fatty acid ester; FFA, free fatty acid; GlcCer, glucosylceramide; LPC, lysophosphatidylcholine; LPE, lysophosphatidylethanolamine; MGDG, monoglycosyldiacylglycerol; NL, neutral lipids; PC, phosphatidylcholine; PL, polar lipids; SG, sterylglycoside; Std, standard;TG, triacylglycerol; TL, total lipids. 


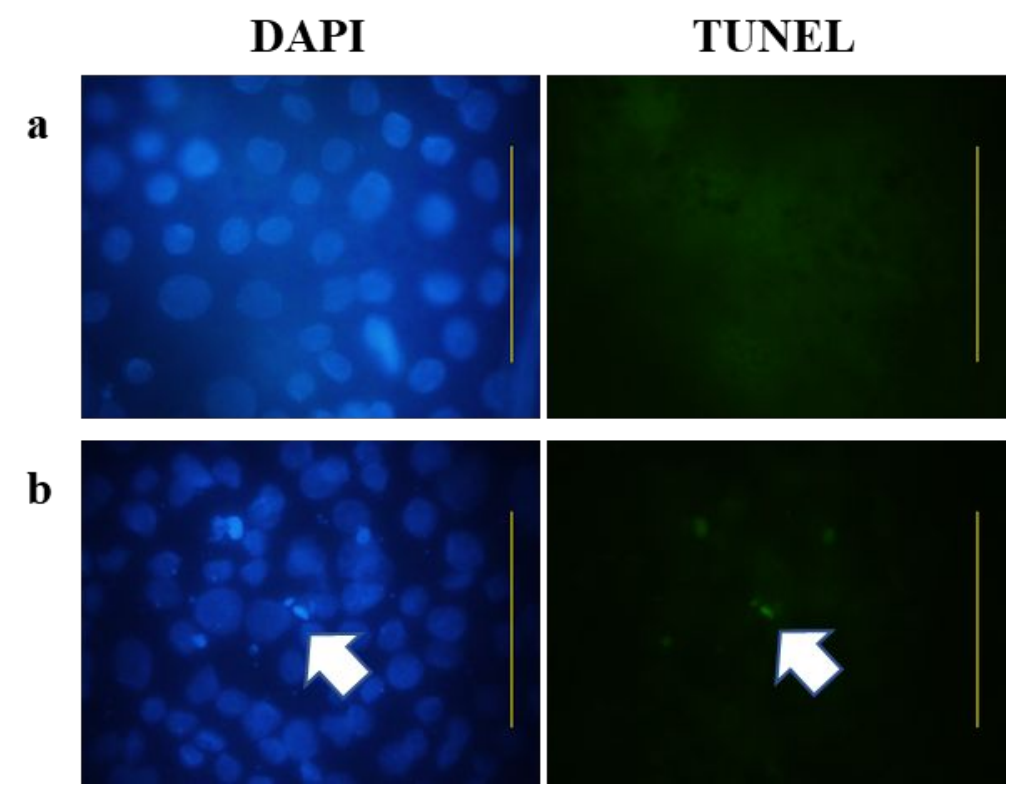

Figure S2. Identification of apoptosis in intestinal cells. Apoptotic cells in Figure 5 were confirmed based on apoptotic body formation and typical chromatin condensation under staining with DAPI and TUNEL. Representative images (objective, 100×). Scale bar: $50 \mu \mathrm{m}$. a, Normal cells (cells treated with lipopolysaccharide and glucosylceramide); b, Apoptotic cells (cells treated with ceramide). 

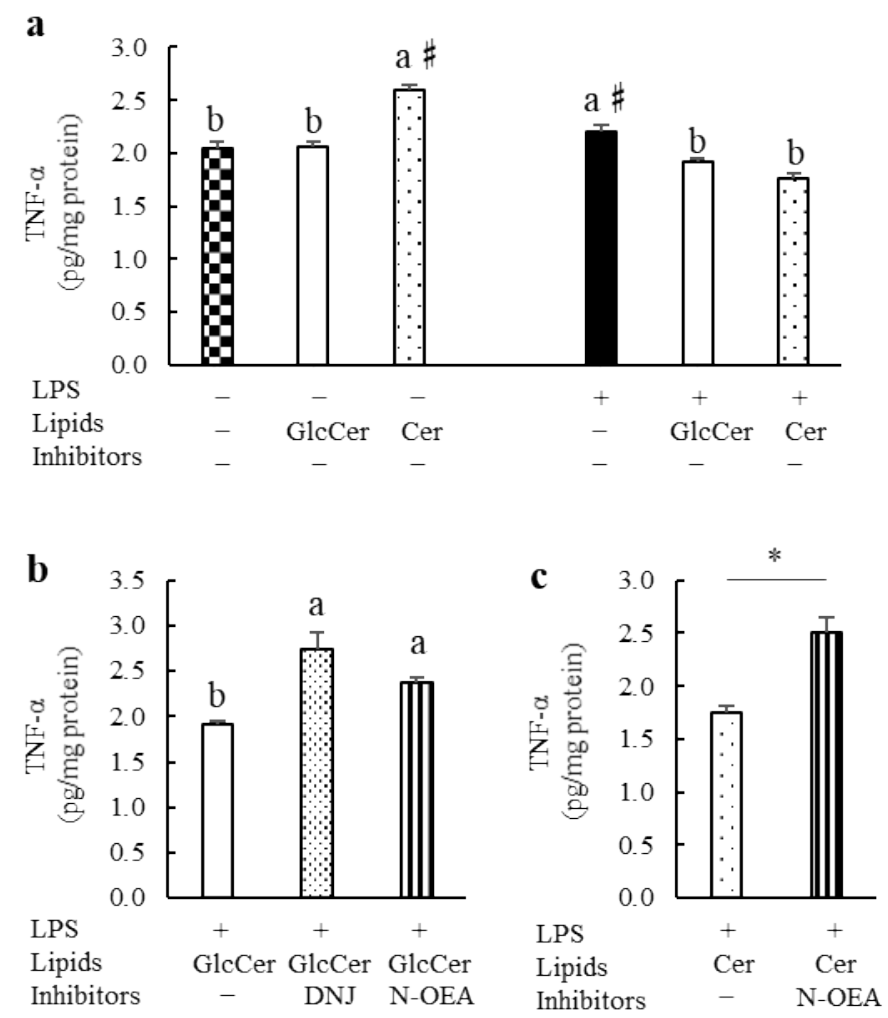

Figure S3. Effects of GlcCer and Cer on LPS-induced TNF- $\alpha$ levels in intestinal cells. Differentiated Caco-2 cells were cultured for $24 \mathrm{~h}$ with sphingolipids $(50 \mu \mathrm{M})$ and LPS $(50 \mu \mathrm{g} / \mathrm{mL})$ along with metabolic inhibitors. TNF- $\alpha$ level in cells was measured using ELISA. a, Cells treated with sphingolipids in the absence and presence of LPS; $\mathbf{b}$, Cells treated with GlcCer and LPS containing glycosylceramidase inhibitor or ceramidase inhibitor; $\mathbf{c}$, Cells treated with Cer and LPS along with a ceramidase inhibitor.

Values represent the mean \pm standard error of mean, $n=6$. Two-way ANOVA (a), one-way ANOVA (b), or Student's $t$-test (c) was performed. Different letters indicate significant differences at $P<0.05$, determined using Tukey's test. Asterisks indicate significant differences between TNF- $\alpha$ secretion of the cells $\left(t\right.$-test, $\left.{ }^{*} P<0.05\right)$. Cer, ceramide; GlcCer, glucosylceramide; LPS, lipopolysaccharide. 